\title{
Cortical hemosiderin is associated with seizures in patients with newly diagnosed malignant brain tumors
}

\author{
Ulrich Roelcke $\cdot$ Larissa Boxheimer • \\ Ali Reza Fathi · Lucia Schwyzer • Marcos Ortega • \\ Jatta Berberat $\cdot$ Luca Remonda
}

Received: 23 May 2013/Accepted: 7 September 2013/Published online: 18 September 2013

(C) Springer Science+Business Media New York 2013

\begin{abstract}
Hemorrhage is common in brain tumors. Due to characteristic magnetic field changes induced by hemosiderin it can be detected using susceptibility weighted MRI (SWI). Its relevance to clinical syndromes is unclear. Here we investigated the patterns of intra-tumoral SWI positivity $\left(\mathrm{SWI}_{\mathrm{pos}}\right)$ as a surrogate for hemosiderin with regard to the prevalence of epilepsy. We report on 105 patients with newly diagnosed supra-tentorial gliomas and brain metastasis. The following parameters were recorded from pre-operative MRI: (1) $\mathrm{SWI}_{\mathrm{pos}}$ defined as dot-like or fine linear signal changes; (2) allocation of SWI $\mathrm{Sos}_{\text {po }}$ to tumor compartments (contrast enhancement, central hypointensity, non-enhancing area outside contrast-enhancement); (3) allocation of $\mathrm{SWI}_{\text {pos }}$ to include the cortex, or $\mathrm{SWI}_{\text {pos }}$ in subcortical tumor parts only; (4) tumor size on T2 weighted and gadolinium-enhanced T1 images. 80 tumors $(76 \%)$ showed $\mathrm{SWI}_{\text {pos }}$ (4/14 diffuse astrocytoma WHO II, 5/9 anaplastic astrocytoma WHO III, 41/46 glioblastoma WHO IV, 30/36 metastasis). The presence of $\mathrm{SWI}_{\text {pos }}$ depended on tumor size but not on patient's age, medication with
\end{abstract}

U. Roelcke $(\bowtie) \cdot$ M. Ortega

Department of Neurology, Neurooncology, Cantonal Hospital, 5001 Aarau, Switzerland

e-mail: roelcke@ksa.ch

U. Roelcke

School of Medicine, University of Basel, 4031 Basel,

Switzerland

L. Boxheimer $\cdot$ J. Berberat $\cdot$ L. Remonda

Department of Neuroradiology, Cantonal Hospital, 5001 Aarau,

Switzerland

A. R. Fathi · L. Schwyzer

Department of Neurosurgery, Cantonal Hospital, 5001 Aarau,

Switzerland antiplatelet drugs or anticoagulation. Seizures occurred in $60 \%$ of patients. Cortical SWI pos significantly correlated with seizures in brain metastasis $(p=0.044)$, and as a trend in glioblastoma $(p=0.062)$. Cortical $\mathrm{SWI}_{\text {pos }}$ may confer a risk for seizures in patients with newly diagnosed brain metastasis and glioblastoma. Whether development of cortical $\mathrm{SWI}_{\text {pos }}$ induced by treatment or by the natural course of tumors also leads to the new onset of seizures has to be addressed in longitudinal studies in larger patient cohorts.

Keywords Brain tumor - Epilepsy · Hemorrhage · Hemosiderin $\cdot$ Magnetic resonance imaging

\section{Introduction}

Intra-tumoral hemorrhage is frequent in brain tumors. Histopathological studies report on an incidence of $13 \%$ in glioblastoma, and of $30 \%$ in oligodendrogliomas [1]. Also brain metastases show hemorrhage which is present in up to $36 \%$ of patients [1]. Acute hemorrhage can result in stroke-like symptoms [1]. On the other hand blood products such as iron and hemosiderin are epileptogenic which has been demonstrated in animal models and patients with vascular malformations [2-4]. Seizures are among the presenting symptoms in $30-50 \%$ of patients with newly diagnosed tumors, and develop in up to $30 \%$ during the post-operative course of disease [5]. A role for hemosiderin in the epileptogenesis of brain tumors has been postulated, however this was not yet addressed in detail [6]. As the above mentioned data on the incidence of intra-tumoral hemorrhage were derived from surgically obtained tissue specimen they do not allow to describe the exact intratumoral localization of hemorrhage [1]. Using 
susceptibility-weighted magnetic resonance imaging (SWI) it is possible to detect signal abnormalities compatible with deposits of blood products such as hemosiderin in vivo (SWI positivity, $\mathrm{SWI}_{\mathrm{pos}}$ ) [7]. This technique therefore enables to characterize $\mathrm{SWI}_{\mathrm{pos}}$ within tumors compartments as well as in the cortical and subcortical brain areas involved by the tumor. The aim of our study was to describe the incidence and distribution of $\mathrm{SWI}_{\text {pos }}$ in newly diagnosed brain tumors, and to assess whether distinct patterns of $\mathrm{SWI}_{\mathrm{pos}}$ relate to the occurence of seizures.

\section{Materials and methods}

\section{Clinical data}

We retrospectively analyzed all successive patients with newly diagnosed supra-tentorial low and high grade glioma and brain metastasis which were treated at our institution between January 2009 and July 2012. As $\mathrm{SWI}_{\text {pos }}$ can be found due to both the presence of blood products and calcification we excluded oligodendroglial tumors where calcification is frequently observed [7]. Patient charts were reviewed with regard to age at diagnosis of brain tumor, gender, and to the presence and semiology of seizures. Medication was recorded with particular emphasis on the administration of platelet inhibitors (aspirin, clopidogrel) or warfarin. The study was approved by the local ethics committee.

\section{MRI data acquisition}

All MR images were obtained as our routine standard diagnostic procedure in patients suspected to suffer from brain tumor using a $1.5 \mathrm{~T}$ Avanto or Espree scanner (Siemens Healthcare, Erlangen, Germany) using an eight channel head array coil. Among our routine MR imaging protocol of the brain which includes contrast- $\left(\right.$ Gadovist $\left.{ }^{\circledR}\right)$ enhanced T1-weighted images an axial SWI sequence was obtained, which is a combination of magnitude and phase images of a 3D velocity compensated gradient echo sequence. The parameters of the T2 SWI were as follows: acquisition time $4: 38 \mathrm{~min}$, slice thickness $2 \mathrm{~mm}$, TE/ $\mathrm{TR}=40 / 40 \mathrm{~ms}, 1$ average and flip angle of $15^{\circ}$.

\section{MRI data analysis}

Pre-operative MRI scans were analyzed. $\mathrm{SWI}_{\text {pos }}$ was defined using the following procedure: (1) the SWI sequences were used to detect dot-like or fine linear hypointense signal changes $\left(\mathrm{SWI}_{\mathrm{pos}}\right)$ [8]; (2) the minimum Intensity Projection (mIP) of the SWI sequences was reviewed to ensure that tumor signal changes are not due to flow signal from vessels; (3) the Phase imaging sequences were used to exclude calcification as a source of intratumoral $\mathrm{SWI}_{\mathrm{pos}}$ [7]. In patients with multiple brain metastases and focal epilepsy the lesion compatible with seizure semiology was chosen for assessment of $\mathrm{SWI}_{\text {pos. }}$. In patients without seizures the largest brain metastasis was chosen.

As there are no established criteria to classify the degree of $\mathrm{SWI}_{\text {pos }}$ we arbitrarily defined three categories: grade $0=$ no $\mathrm{SWI}_{\text {pos }}$, grade $\mathrm{I}=$ one to five focal signal changes (either dot-like or fine linear or both), grade II $=\geq$ six focal signal changes [8].

$\mathrm{SWI}_{\text {pos }}$ was also characterized by its cortical and/or subcortical localization. In patients with contrast-enhancing tumors we further assessed whether $\mathrm{SWI}_{\mathrm{pos}}$ involved the enhancing tumor area, the central hypointensity within, or the non-enhancing area outside contrast-enhancement.

Tumor size was measured on axial contrast-enhanced T1 and $\mathrm{T} 2$ weighted images according to the RANO criteria [9]. The two maximal perpendicular diameters were calculated on the plane showing the largest tumor diameter. In patients with multiple brain metastases the lesion which corresponded to the focal seizure semiology was chosen. In patients without seizures the largest lesion was chosen.

\section{Statistics}

We tested whether $\mathrm{SWI}_{\text {pos }}$ was associated with age, treatment with platelet inhibitors or warfarin, or with tumor size. For comparison of categorial or continuous variables the Fisher's exact and Chi square test was applied. We used regression analysis to estimate whether the presence of seizures was associated with any of the clinical characteristics and imaging results. Statistical analyses were performed using SigmaStat 12 Software, SyStat, Inc., USA.

\section{Results}

We identified 105 patients (68 male, 37 female, age $59.5 \pm 12.3$ years (mean $\pm \mathrm{SD}$ ) with newly diagnosed brain tumors (14 low-grade glioma WHO II, 9 anaplastic glioma WHO III, 46 glioblastoma WHO IV, 36 brain metastasis) (Table 1). The histopathological diagnosis was established in $91 \%$ by partial or gross total resection, $9 \%$ of patients were biopsied only. $87 \%$ of patients with brain metastasis suffered from multiple lesions. 25 patients (24\%) received platelet inhibitors or warfarin for cardiovascular disorders. All glioblastomas and brain metastases showed blood-brain barrier (BBB) disruption to a variable degree as evident on pre-operative gadolinium-enhanced MRI. Two low-grade gliomas and five anaplastic gliomas showed circumscribed BBB disruption. 
Table 1 Clinical data and imaging results

\begin{tabular}{|c|c|c|c|c|c|c|c|}
\hline Histology & $\mathrm{n}$ & $\begin{array}{l}\text { Age }^{\mathrm{a}} \\
\text { years }\end{array}$ & $\begin{array}{l}\text { PI } \\
\text { Warf } \\
\%\end{array}$ & $\begin{array}{l}\text { Tumor size } \\
\left(\mathrm{T} 1+\mathrm{CE}^{\mathrm{b}}\right)^{\mathrm{a}} \\
\mathrm{mm}^{2}\end{array}$ & $\begin{array}{l}\text { Tumor size } \\
\left(\mathrm{T} 2^{\mathrm{b}}\right)^{\mathrm{a}} \\
\mathrm{mm}^{2}\end{array}$ & $\begin{array}{l}\mathrm{SWI}_{\mathrm{pos}} \\
\text { tumors } \\
\mathrm{n}(\%)\end{array}$ & $\begin{array}{l}\mathrm{SWI}_{\text {pos }} \text { located } \\
\text { in } \mathrm{BBBD}^{\mathrm{d}} \\
\%\end{array}$ \\
\hline Low-grade glioma & 14 & $53 \pm 12$ & 14 & $3.1 \pm 1.6$ & $20.5 \pm 14.1$ & $4(29)$ & 50 \\
\hline Anaplastic astrocytoma & 9 & $58 \pm 15$ & 44 & $4.8 \pm 8.4$ & $21.2 \pm 11.0$ & $5(56)$ & 100 \\
\hline Glioblastoma & 46 & $59 \pm 12$ & 15 & $13.6 \pm 8.6$ & $29.9 \pm 15.7$ & $42(91)$ & 90 \\
\hline Brain metastasis ${ }^{\mathrm{c}}$ & 36 & $63 \pm 11$ & 33 & $7.5 \pm 8.0$ & $19.5 \pm 12.9$ & $29(83)$ & 97 \\
\hline
\end{tabular}

a Data are presented as mean \pm SD. PI, Warf: percentage of patients receiving platelet inhibitors or warfarin

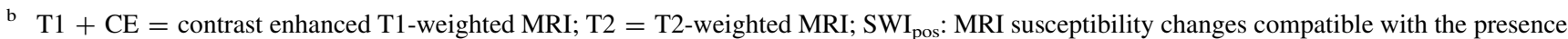
of hemosiderin (see "Materials and methods" section)

c Primary tumor: small cell lung cancer $(n=4)$, non-small cell lung cancer $(n=13)$, breast cancer $(n=5)$, malignant melanoma $(n=4)$, prostate $(n=2)$, urothel $(n=2)$, colon $(n=1)$, esophagus $(n=1)$, uterus $(n=1)$, unknown $(n=3)$

d BBBD: tumor area with blood-brain barrier disruption and/or central hypointensity
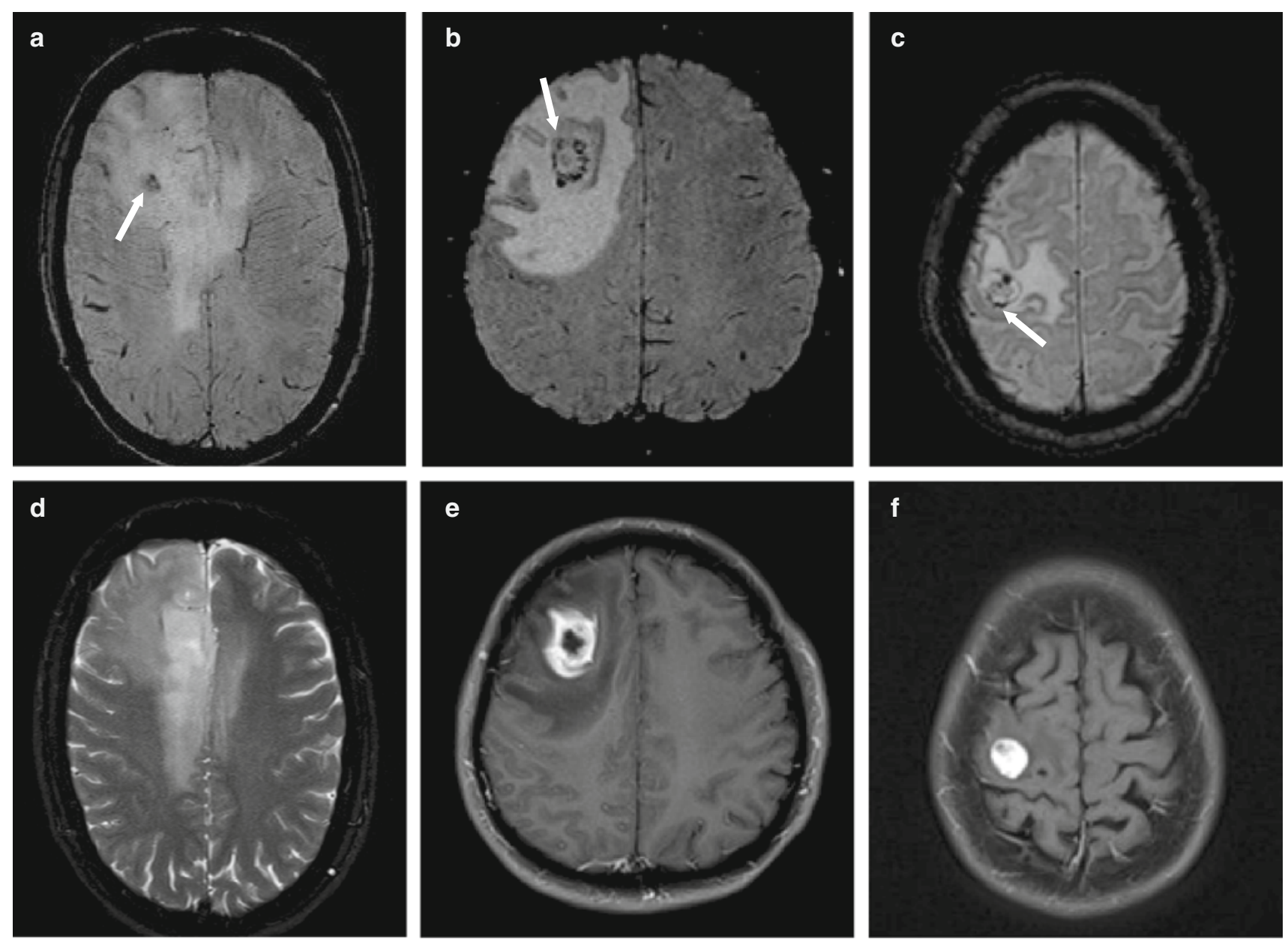

Fig. 1 Distribution of hemosiderin in newly diagnosed brain tumors. Fibrillary astrocytoma (left), glioblastoma (middle), brain metastasis

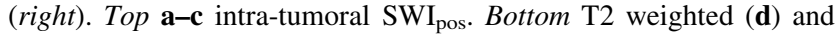

Frequency and distribution of $\mathrm{SWI}_{\text {pos }}$

$\mathrm{SWI}_{\text {pos }}$ was observed in 80 patients $(76 \%)$ and was significantly higher in glioblastoma and brain metastasis gadolinium enhanced T1 weighted (e, f) MRI. Arrow subcortical (a, b) and cortical (c) $\mathrm{SWI}_{\text {pos }}$

compared to the other subgroups (Chi square 9.455, $p=0.024$ ) (Table 1). The distribution of $\mathrm{SWI}_{\text {pos }}$ within the tumors and with regard to subcortical location and cortical involvement is illustrated in Fig. 1. In the majority 
of patients with malignant tumors $\mathrm{SWI}_{\text {pos }}$ was located to the area of BBB disruption or the central hypointensity and

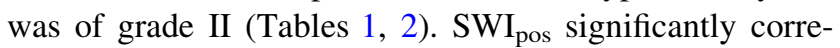
lated with tumor size on $\mathrm{T} 2$ weighted and gadolinium enhanced T1 MRI $(p<0.001)$, but neither with patient's age $(p=0.371)$ nor with treatment with platelet inhibitors or warfarin $(p=0.290)$.

Seizures, tumor size and $\mathrm{SWI}_{\text {pos }}$

For the whole group of 105 patients the presence of seizures correlated with tumor size on MRI T2 and gadolinium enhanced T1 weighted MR ( $p=0.009,0.002)$, but not with age $(p=0.127)$ and medication with platelet inhibitors or warfarin $(p=0.423)$. SWI $\mathrm{Sos}_{\text {sevealed a significant }}$ correlation with seizures as onset symptoms $(p=0.005$, likelihood ratio 0.62 ), but with moderate sensitivity (0.5) and specificity (0.2) (Fisher's exact test). A stronger association with seizures was observed when $\mathrm{SWI}_{\text {pos }}$ involved the cerebral cortex $(p=0.003$, likelihood ratio 2.2). Here we found a sensitivity of 0.67 and a specificity of 0.7 . Involvement of the cortex by tumor alone was of marginal significance $(p=0.051)$.

$\mathrm{SWI}_{\text {pos }}$ with regard to seizures in the histological subgroups is detailed in Table 2 and is summarized in Fig. 2. Seizure frequency and cortical involvement by tumor was highest in low-grade glioma, but cortical $\mathrm{SWI}_{\text {pos }}$ was lowest in this subgroup (Fig. 2). Vice versa 95 and $77 \%$ of patients with glioblastoma and brain metastasis showed cortical involvement by tumor, but only 65 and $73 \%$ showed cortical SWI $\mathrm{SWo}_{\text {po }}$ and 43 and $72 \%$ of these patients experienced seizures. Testing all clinical and imaging variables we observed a significant correlation between cortical SWI $\mathrm{Sos}_{\mathrm{p}}$ and the presence of seizures for brain metastasis $(p=0.044)$, and a trend for glioblastoma $(p=0.062)$. Tumor $\mathrm{SWI}_{\text {pos }}$ per se did not correlate with the presence of seizures. Logistic regression analysis was not possible for

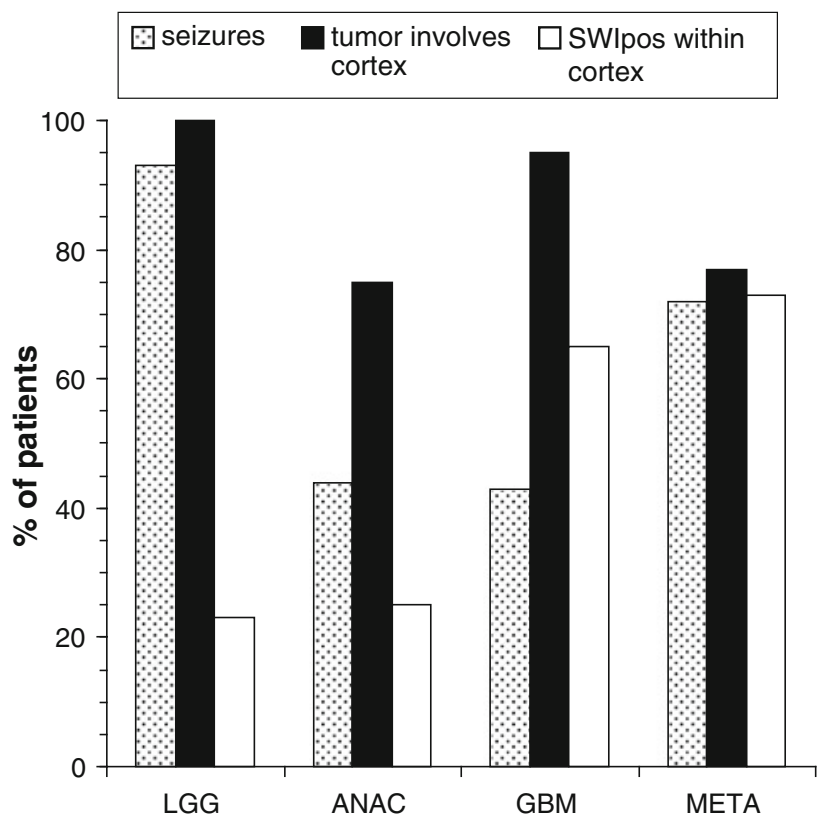

Fig. 2 Frequency of seizures, cortical involvement by tumor and

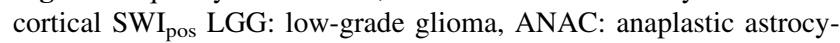
toma, GBM: glioblastoma, META: brain metastasis

low-grade glioma and anaplastic astrocytoma due to the small number of patients in these subgroups. The Chi square test showed no correlation between the grade of $\mathrm{SWI}_{\text {pos }}$ and the presence of seizures in all subgroups (Table 2).

\section{Discussion}

Our data indicate that intra-tumoral hemorrhage as studied with susceptibility weighted MRI ( $\left.\mathrm{SWI}_{\text {pos }}\right)$ occurs more frequent than estimated from histopathological analysis [1]. This difference is most likely explained by sampling errors in patients who underwent partial resection or biopsy only [1]. In line with earlier reports the incidence of $\mathrm{SWI}_{\mathrm{pos}}$

Table $2 \mathrm{SWI}_{\mathrm{pos}}$ and seizures

\begin{tabular}{|c|c|c|c|c|c|c|}
\hline \multirow{2}{*}{$\begin{array}{l}\text { Histology } \\
\text { Low-grade glioma }\end{array}$} & \multicolumn{2}{|c|}{$\begin{array}{l}\text { Presence } \\
\text { of seizures } \\
\mathrm{n}(\%)\end{array}$} & \multirow{2}{*}{$\begin{array}{l}\mathrm{SWI}_{\text {pos }} \text { in tumor } \\
\text { subgroups } \\
\text { n }(\%)\end{array}$} & \multirow{2}{*}{$\begin{array}{l}\text { Grade of } \\
\operatorname{SWI}_{\text {pos }}(0 / 1 / 2)^{\mathrm{a}} \\
\mathrm{n}\end{array}$} & \multirow{2}{*}{$\begin{array}{l}\text { Tumor involves } \\
\text { cortex } \\
\mathrm{n}(\%)\end{array}$} & \multirow{2}{*}{$\begin{array}{l}\text { Tumors with } \\
\text { cortical SWI } \\
\text { n }(\%)\end{array}$} \\
\hline & Yes & $13(93)$ & & & & \\
\hline & No & $1(7)$ & $0(0)$ & $1 / 0 / 0$ & $0(0)$ & $0(0)$ \\
\hline \multirow[t]{2}{*}{ Anaplastic astrocytoma } & Yes & $4(44)$ & $1(25)$ & $3 / 1 / 0$ & $3(75)$ & $1(25)$ \\
\hline & No & $5(56)$ & $4(80)$ & $1 / 1 / 3$ & $5(100)$ & $2(40)$ \\
\hline \multirow[t]{2}{*}{ Glioblastoma } & Yes & $20(43)$ & $16(85)$ & $4 / 0 / 16$ & $19(95)$ & $13(65)$ \\
\hline & No & $26(57)$ & $25(96)$ & $1 / 1 / 24$ & $24(92)$ & $11(42)$ \\
\hline \multirow[t]{2}{*}{ Brain metastasis } & Yes & $26(72)$ & $21(81)$ & $5 / 5 / 16$ & $20(77)$ & $19(73)$ \\
\hline & No & $10(28)$ & $9(90)$ & $1 / 0 / 9$ & $9(90)$ & $4(40)$ \\
\hline
\end{tabular}

a The grade of $\mathrm{SWI}_{\text {pos }}$ did not correlate with seizures (low-grade glioma $p=0.422$, anaplastic astrocytoma $p=0.283$, glioblastoma $p=0.159$, brain metastasis $p=0.209$; Chi square test) 
exceeded $80 \%$ in patients with glioblastoma and brain metastasis, and was low in patients with low-grade glioma $[8,10]$. Therefore the presence of $\mathrm{SWI}_{\mathrm{pos}}$ on MRI may serve as a non-invasive marker of tumor malignancy. As the majority of patients with glioblastoma and brain metastasis show $\mathrm{SWI}_{\text {pos }}$ within the area of BBB disruption or the central tumor core it is likely that $\mathrm{SWI}_{\text {pos }}$ represents a non-specific marker of vascular leakage common to malignant brain tumors. The abnormal vascular structure in malignant gliomas is composed by immature and retiform capillaries as well as by thin walled sinusoids which may predispose to hemorrhage [11]. The origin of $\mathrm{SWI}_{\text {pos }}$ in non-enhancing low grade glioma is unclear. It may be hypothesized that circumscribed $\mathrm{SWI}_{\text {pos }}$ lesions (Fig. 1a) represent malignant tumor foci, and that the detection of new or enlarging $\mathrm{SWI}_{\mathrm{pos}}$ during the long-term course of low-grade tumors indicates subsequent transformation into malignant glioma. Biopsy of SWI $\mathrm{S}_{\text {pos }}$ tumor regions aiming at the detection of malignant glioma may unravel vascular changes within these lesions.

The overall clinical impact of hemorrhage in brain tumor patients has not been addressed in detail. Hemorrhage with stroke-like symptoms is rare and occurs in 2 to $3 \%$ of patients $[1,12]$. These clinical events are clearly less frequent than the histopathological detection of hemosiderin or $\mathrm{SWI}_{\text {pos }}$ on MRI (our data) [1, 11]. Small intratumoral hemorrhage which does not result in stroke-like symptoms may be present for a hitherto unknown period of time. The evolution of the characteristic signal changes due to hemorrhage has been studied in $\mathrm{T} 1$ and $\mathrm{T} 2$ weighted MRI in various conditions including brain tumors [13, 14]. Hemorrhage induced hypointensity on $\mathrm{T} 2$ weighted sequences can be already observed 1 day after bleeding. As SWI are derived from $\mathrm{T} 2$ weighted sequences a similar behaviour of intra-tumoral $\mathrm{SWI}_{\text {pos }}$ can be assumed. Intratumoral hemorrhage at the time of tumor detection may therefore be present for several days or even longer. Whether single or multiple small intra-tumoral hemorrhages contribute to persisting or increasing neurological dysfunction as e.g. in patients with amyloid angiopathy cannot be answered at the present time [15]. However cortical $\mathrm{SWI}_{\text {pos }}$ in our study was significantly associated with the presence of seizures in brain metastasis, and as a trend in glioblastoma. This would be in line with studies in animals and patients with vascular malformations. Intracortical injection of hemoglobin or ferric chloride in rats leads to persisting spike activity and seizures which occur 2 to 8 days after injection $[2,3]$. In patients with resected cavernoma areas with hemosiderin deposits show neuronal loss, glial proliferation and upregulation of NMDA receptor subunits to a magnitude considered to be able to promote seizures [16]. Accordingly cortical $\mathrm{SWI}_{\text {pos }}$ may indicate a role for hemosiderin in the epileptogenicity of glioblastoma and brain metastasis. It has been proposed that disruption of the BBB per se induces seizures by extravasation of albumine and activation of transforming growth factor beta (TGF $\beta$ ) [17]. Whether this assumption is relevant to patients with brain tumors has not been investigated. In our series all patients with glioblastoma or brain metastasis showed BBB disruption, but only 43 and $72 \%$ respectively experienced seizures. Our findings therefore do not support the view that BBB disruption or cortical involvement alone explain the epileptogenicity of these brain tumors.

Cortical tumor location represented a strong factor associated with pre-operative seizures in a series of 508 patients with low-grade glioma [18]. In line with this finding we found in our small subgroup of low-grade glioma patients a high percentage of cortical involvement, but only few tumors with $\mathrm{SWI}_{\text {pos. }}$. Therefore biochemical alterations other than hemosiderin deposits may account for the occurrence of seizures in these tumors. In general changes in $\mathrm{pH}$, amino acid composition, NMDA receptor status have been discussed to be involved in epileptogenesis [6]. However, all these metabolic changes cannot be assessed in a non-invasive manner in human patients. Therefore they cannot serve as in vivo markers to determine the risk for seizures. From a methodological point of view it can be assumed that $\mathrm{SWI}_{\mathrm{pos}}$ in fact reflects hemosiderin if $\mathrm{mIP}$ and Phase images exclude tumor calcification and flow signal. However, prospective tissue validation of $\mathrm{SWI}_{\mathrm{pos}}$ in brain tumors is required. Preliminary data of our group obtained from a retrospective series indicate a high specificity to identify hemosiderin using susceptibility weighted MRI [19].

The limitation of our study is the small number of patients, particularly of low-grade gliomas and anaplastic astrocytomas, which however reflects their incidence in the general population and therefore in our clinical routine. Cortical SWI $\mathrm{Sps}_{\text {ps }}$ an independent predictor of subsequent seizures should lead to re-evaluate the use of prophylactic anticonvulsants in patients showing this feature. From our results we propose to longitudinally investigate the presence and evolution of $\mathrm{SWI}_{\text {pos }}$ with particular focus on glioblastoma and brain metastasis in larger patient cohorts. Several issues may contribute to hemosiderin deposits during the course of brain tumors. $\mathrm{SWI}_{\mathrm{pos}}$ is often detected after brain tumor surgery. Irradiation can result in vascular leakage and microbleeds [20, 21]. In addition hemorrhage may also result from anti-angiogenic therapy, uncontrolled tumor growth and from the appearance of new brain metastasis. In a recent study on patients with newly diagnosed brain metastasis from malignant melanoma $94 \%$ of lesions involved the cerebral cortex, and $54 \%$ were hemorrhagic [22]. In those patients who initially did not present with seizures the risk to develop seizures within three 
months after the time of the diagnosis was $17 \%$ if no prophylactic anticonvulsants were administered. In contrast no patient treated with prophylactic anticonvulsants suffered from seizures during that period. It appears worthwhile to prospectively assess whether cortical $\mathrm{SWI}_{\mathrm{pos}}$ represents a risk factor for the occurrence of seizures, and whether cortical $\mathrm{SWI}_{\text {pos }}$ determines the risk for seizure relapse once anticonvulsants are discontinued after surgery.

Acknowledgments The authors thank Professor Rainer Grobholz, Dept. Pathology, Cantonal Hospital Aarau, for sharing his expertise and discussion on intra-tumoral hemorrhage and manuscript reading.

Conflict of interest The authors declare that they have no conflict of interest.

Ethical standard The study complies with the current laws and was approved by the Ethics Committee of the Cantonal Hospital, Aarau, Switzerland.

\section{References}

1. Kondziolka D, Bernstein M, Resch L, Tator CH, Fleming JF, Vanderlinden RG, Schutz H (1987) Significance of hemorrhage into brain tumors: clinicopathological study. J Neurosurg 67: 852-857

2. Rosen AD, Frumin NV (1979) Focal epileptogenesis after intracortical hemoglobin injection. Exp Neurol 66:277-284

3. Ueda Y, Willmore LJ, Triggs WJ (1998) Amygdalar injection of $\mathrm{FeCl} 3$ causes spontaneous recurrent seizures. Exp Neurol 153: 123-127

4. Moran NF, Fish DR, Kitchen N, Shorvon S, Kendall BE, Stevens JM (1999) Supratentorial cavernous haemangiomas and epilepsy: a review of the literature and case series. J Neurol Neurosurg Psychiatry 66:561-568

5. van Breemen MS, Wilms EB, Vecht CJ (2007) Epilepsy in patients with brain tumours: epidemiology, mechanisms, and management. Lancet Neurol 6:421-430

6. Beaumont A, Whittle IR (2000) The pathogenesis of tumour associated epilepsy. Acta Neurochir (Wien) 142:1-15

7. Robinson RJ, Bhuta S (2011) Susceptibility-weighted imaging of the brain: current utility and potential applications. J Neuroimaging 21:e189-e204

8. Park MJ, Kim HS, Jahng GH, Ryu CW, Park SM, Kim SY (2009) Semiquantitative assessment of intra-tumoral susceptibility signals using non-contrast-enhanced high-field high-resolution susceptibility-weighted imaging in patients with gliomas: comparison with MR perfusion imaging. AJNR Am J Neuroradiol 30:1402-1408

9. Wen PY, Macdonald DR, Reardon DA et al (2010) Updated response assessment criteria for high-grade gliomas: response assessment in neuro-oncology working group. J Clin Oncol 28:1963-1972

10. Li C, Ai B, Li Y, Qi H, Wu L (2010) Susceptibility-weighted imaging in grading brain astrocytomas. Eur J Radiol 75:e81-e85

11. Liwnicz BH, Wu SZ, Tew JM Jr (1987) The relationship between the capillary structure and hemorrhage in gliomas. J Neurosurg 66:536-541

12. Kothbauer P, Jellinger K, Falment H (1979) Primary brain tumour presenting as spontaneous intracerebral haemorrhage. Acta Neurochir (Wien) 49:35-45

13. Bradley WG Jr (1993) MR appearance of hemorrhage in the brain. Radiology 189:15-26

14. Allkemper T, Tombach B, Schwindt W, Kugel H, Schilling M, Debus O, Möllmann F, Heindel W (2004) Acute and subacute intracerebral hemorrhages: comparison of MR imaging at 1.5 and 3.0 T-initial experience. Radiology 232:874-881

15. Werring DJ, Frazer DW, Coward LJ, Losseff NA, Watt H, Cipolotti L, Brown MM, Jäger HR (2004) Cognitive dysfunction in patients with cerebral microbleeds on $\mathrm{T} 2 *$-weighted gradientecho MRI. Brain 127:2265-2275

16. Kamida T, Takeda Y, Fujiki M, Abe T, Abe E, Kobayashi H (2007) Nitric oxide synthase and NMDA receptor expressions in cavernoma tissues with epileptogenesis. Acta Neurol Scand 116:368-373

17. Friedman A, Kaufer D, Heinemann U (2009) Blood-brain barrier breakdown-inducing astrocytic transformation: novel targets for the prevention of epilepsy. Epilepsy Res 85:142-149

18. You G, Sha Z-Y, Yan W et al (2012) Seizure characteristics and outcomes in 508 Chinese adult patients undergoing primary resection of low-grade gliomas: a clinicopathological study. Neuro-Oncology 14:230-241

19. Berberat J, Grobholz R, Boxheimer L, Remonda L, Roelcke U (2012) Differentiating calcification and hemorrhage in tumor tissue using susceptibility weighted imaging [abstract]. Proc Int Soc Magn Res Med 20:3197

20. Noyce AJ, McCrae S, Gawler J, Evanson J (2010) Teaching neuroImages: microhemorrhages resulting from cranial radiotherapy in childhood. Neurology 75:e2-e3

21. Zeng QS, Kang XS, Li CF, Zhou GY (2011) Detection of hemorrhagic hypointense foci in radiation injury region using susceptibility-weighted imaging. Acta Radiol 52:115-119

22. Goldlust SA, Hsu M, Lassman AB, Panageas KS, Avila EK (2012) Seizure prophylaxis and melanoma brain metastases. J Neurooncol 108:109-114 